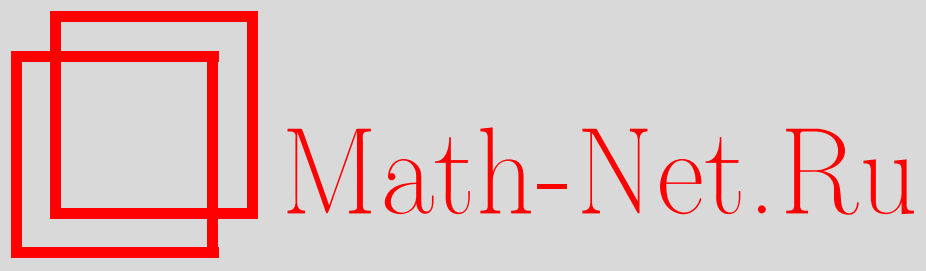

Т. Оувада, Интерполяционная теорема Сарасона для аналитических скрещенных произведений, Функи. анализ и его прил., 2006, том 40, выпуск 2, 82-86

DOI: https://doi.org/10.4213/faa11

Использование Общероссийского математического портала Math-Net.Ru подразумевает, что вы прочитали и согласны с пользовательским соглашением

http://www .mathnet.ru/rus/agreement

Параметры загрузки:

IP : 54.198 .64 .247

26 апреля 2023 г., 11:58:02

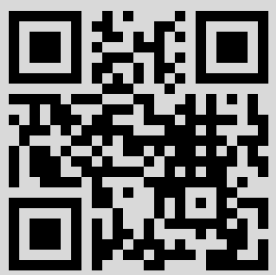




\title{
Интерполяционная теорема Сарасона для аналитических скрещенных произведений ${ }^{*}$
}

\author{
(C) 2006. Т. ОувАДА
}

Сарасон [7] исследовал теорию обобщенной интерполяции в алгебре Харди $\mathbb{H}^{\infty}(\mathbb{T})$ над единичной окружностью и доказал интерполяционную теорему. Рассматривая аналитические скрещенные произведения как «некоммутативные алгебры Харди», Сайто [6] ввел понятие интерполяции в аналитических скрещенных произведениях и доказал интерполяционную теорему Сарасона для случая аналитического скрещенного произведения, определяемого конечной алгеброй фон Неймана и ее автоморфизмом [6, Theorem 3.2]. Его метод доказательства таков, что избавиться в нем от предположения о конечности алгебр фон Неймана, по-видимому, нельзя. Хотя Иосино [8] и дал простое доказательство интерполяционной теоремы Сарасона в $\mathbb{H}^{\infty}(\mathbb{T})$, но оно опирается на условие, что оператор, сопряженный к оператору Ганкеля, снова должен быть оператором Ганкеля. Разумеется, это не гарантировано в общей ситуации аналитических скрещенных произведений (ср. [4]). Чтобы провести аналог подхода Иосино, мы сформулируем условие, эквивалентное условию из теоремы Нехари [2, Theorem 3.5]. Затем мы докажем, что интерполяционная теорема Сарасона в формулировке Сайто верна и без предположения о конечности соответствующей алгебры фон Неймана.

Пусть $M$ - алгебра фон Неймана, а $\alpha-$ ее $*$-автоморфизм. Мы считаем, что $M$ действует на некоммутативном $L^{2}$-пространстве $L^{2}(M)$ в смысле Хагерупа (cp. [1]). Если $x \in M$, то обозначим через $\ell_{x}$ (соответственно $r_{x}$ ) оператор в $L^{2}(M)$, действующий по формуле $\ell_{x} y=x y$ (соответственно $r_{x} y=y x$ ), $y \in L^{2}(M)$. Тогда $\ell$ (соответственно $r$ ) представляет собой точное нормальное представление (соответственно антипредставление) алгебры $M$ в гильбертовом пространстве $L^{2}(M)$. Положим

$$
\ell(M)=\left\{\ell_{x} \mid x \in M\right\} \quad \text { и } \quad r(M)=\left\{r_{x} \mid x \in M\right\} .
$$

Оператор $J$, заданный на $L^{2}(M)$ формулой $J y=y^{*}, y \in L^{2}(M)$, представляет собой сопряженно-линейную изометрическую инволюцию в $L^{2}(M)$. Пусть $L^{2}(M)_{+}-$положительная часть пространства $L^{2}(M)$. Поскольку четверка $\left\{\ell(M), L^{2}(M), J, L^{2}(M)_{+}\right\}$является стандартной формой алгебры $M$ в смысле Хагерупа [1], то алгебры фон Неймана $\ell(M)$ и $r(M)$ суть коммутанты друг друга и $J \ell(M) J=r(M)$. Более того, согласно [1, Theorem 3.2], существует такой унитарный оператор $u$ в $L^{2}(M)$, что $\ell_{\alpha(x)}=u \ell_{x} u^{*}$ и $r_{\alpha(x)}=u r_{x} u^{*}, x \in M$. Чтобы построить скрещенное произведение, рассмотрим гильбертово пространство

$$
\mathbb{L}^{2}=\left\{f: \mathbb{Z} \rightarrow L^{2}(M) \mid \sum_{n \in \mathbb{Z}}\|f(n)\|_{2}^{2}<\infty\right\},
$$

*Работа частично поддержана субсидией на научные исследования, выделенной Министерством образования, культуры, спорта, науки и техники Японии. 
где $\|\cdot\|_{2}$ - норма в $L^{2}(M)$. Для любого $x \in M$ определим операторы $L_{x}, R_{x}$, $L_{\delta}$ и $R_{\delta}$ в $\mathbb{L}^{2}$ формулами

$$
\begin{array}{llrl}
\left(L_{x} f\right)(n) & =\ell_{x} f(n), & & \left(R_{x} f\right)(n)=r_{\alpha^{n}(x)} f(n), \\
\left(L_{\delta} f\right)(n) & =u f(n-1), & & \left(R_{\delta} f\right)(n)=f(n-1),
\end{array}
$$

где $f \in \mathbb{L}^{2}$ и $n \in \mathbb{Z}$. Пусть $L(M)=\left\{L_{x} \mid x \in M\right\}$ и $R(M)=\left\{R_{x} \mid x \in M\right\}$. Положим $\mathfrak{L}=\left\{L(M), L_{\delta}\right\}^{\prime \prime}$ and $\mathfrak{R}=\left\{R(M), R_{\delta}\right\}^{\prime \prime}$ и определим левое (правое) аналитическое скрещенное произведение $\mathfrak{L}_{+}$(соответственно $\mathfrak{R}_{+}$) как $\sigma$-слабо замкнутую подалгебру в $\mathfrak{L}$ (соответственно в $\mathfrak{R})$, порожденную $L(M)$ и $L_{\delta}$ (соответственно $R(M)$ и $\left.R_{\delta}\right)$. Далее, положим

$$
\mathbb{H}^{2}=\left\{f \in \mathbb{L}^{2} \mid f(n)=0, n<0\right\} .
$$

Заметим, что $\mathfrak{L}_{+}=\left\{T \in \mathfrak{L} \mid T \mathbb{H}^{2} \subseteq \mathbb{H}^{2}\right\}$. Теперь определим операторы Тёплица и Ганкеля следующим образом. Пусть $P$ - ортогональный проектор на $\mathbb{H}^{2}$ в $\mathbb{L}^{2}$. Если $A$ принадлежит $\mathfrak{L}$, то оператор Теплица $T_{A}$ и оператор Ганкеля $H_{A}$ определены на $\mathbb{H}^{2}$ соотношениями

$$
T_{A} f=P(A f) \quad \text { и } \quad H_{A} f=W(I-P)(A f) \quad\left(\forall f \in \mathbb{H}^{2}\right),
$$

где $W$ - унитарный оператор в $\mathbb{L}^{2}$, действующий по формуле $(W f)(n)=$ $f(-n-1)$ для всех $n \in \mathbb{Z}$. Заметим, что $W^{2}=I$ и $W P W=I-P$. Мы отсылаем читателя к $[3,5,6]$ за обсуждением этих алгебр и операторов, включая некоторые их элементарные свойства. Будем говорить, что замкнутое подпространство $\mathfrak{M}$ в $\mathbb{L}^{2}$ двусторонне инвариантно, если $\mathfrak{L}_{+} \mathfrak{M} \subseteq \mathfrak{M}$ и $\mathfrak{R}_{+} \mathfrak{M} \subseteq \mathfrak{M}$. Теперь рассмотрим двустороннее инвариантное подпространство в $\mathbb{H}^{2}$ вида $T_{V} \mathbb{H}^{2}$ для некоторого унитарного оператора $V$ из $\mathfrak{L}_{+}$. Положим $\mathscr{K}=\mathbb{H}^{2} \ominus T_{V} \mathbb{H}^{2}$. Ортогональный проектор в $\mathbb{L}^{2}$ с областью значений $\mathscr{K}$ обозначим через $P_{\mathscr{K}}$. Для любого линейного ограниченного оператора $A$ в $\mathscr{K}$ определим оператор $S_{A}$ в $\mathscr{K}$ формулой $S_{A} f=P_{\mathscr{K}}(A f)$ (для всех $f \in \mathscr{K}$ ). Если линейный ограниченный оператор $A$ в $\mathscr{K}$ может быть записан в виде $S_{C}$ для некоторого оператора $C$ из $\mathfrak{L}_{+}$, то будем говорить, что оператор $C$ интерполирует оператор $A$.

Целью заметки является доказательство следующей теоремы.

Tеорема 1 (ср. [6, Theorem 3.2]). Если $A$ - линейный ограниченный оператор в $\mathscr{K}$, коммутирующий с $P_{\mathscr{K}} \mathfrak{R}_{+} P_{\mathscr{K}}$, то существует оператор $C$ из $\mathfrak{L}_{+}$, интерполирующий оператор $A\left(m\right.$. е. удовлетворяющий условию $\left.A=S_{C}\right)$, такой, что $\|A\|=\|C\|$. Таким образом, $\left(P_{\mathscr{K}} \mathfrak{L}_{+} P_{\mathscr{K}}\right)^{\prime}=P_{\mathscr{K}} \mathfrak{R}_{+} P_{\mathscr{K}}$ u $\left(P_{\mathscr{K}} \mathfrak{R}_{+} P_{\mathscr{K}}\right)^{\prime}$ $=P_{\mathscr{K}} \mathfrak{L}_{+} P_{\mathscr{K}}$.

Для доказательства нам нужна некоторая подготовка. Сайто [5] доказал следующие полезные результаты.

ПРЕДЛОЖЕНИЕ 2 [5, Proposition 2.2]. Пусть $T$ - линейный ограниченный оператор в $\mathbb{L}^{2}$. Отождествим $\mathbb{L}^{2}$ с тензорным произведением $\ell^{2}(\mathbb{Z}) \otimes L^{2}(M)$ пространств $\ell^{2}(\mathbb{Z})$ и $L^{2}(M)$. Тогда

(i) оператор $T$ принадлежит коммутанту алгебры $L(M)$ в том и только в том случае, когда он имеет матричное представление вида $\left(r_{x_{i j}}\right)_{i, j=-\infty}^{\infty}$, где $x_{i j} \in M$ для всех $i, j \in \mathbb{Z}$;

(ii) оператор $T$ принадлежит коммутанту алгебры $R(M)$ в том и только в том случае, когда он имеет матричное представление вида $\left(l_{x_{i j}} u^{i-j}\right)_{i, j=-\infty}^{\infty}$, где $x_{i j} \in M$ для всех $i, j \in \mathbb{Z}$. 
Для любого $A$ из $\mathfrak{L}$ положим $\widetilde{H}_{W A W}=W(I-P)(W A W) P$. Отметим, что вообще говоря, $\widetilde{H}_{W A W}$ не является оператором Ганкеля, поскольку $W A W$ не всегда лежит в $\mathfrak{L}$. Действительно, заметим, что оператор $\widetilde{H}_{W A W}$ является оператором Ганкеля для любого $A \in \mathfrak{L}$ тогда и только тогда, когда $\alpha^{2}$ тривиально на $M$. Поэтому обычно нужно различать $\widetilde{H}_{W A W}$ и $H_{W A W}$. Отметим при этом, что если $A \in \mathfrak{L}$, то $\left(H_{A}\right)^{*}=\widetilde{H}_{W A^{*} W}$.

Имина и Сайто [2] в связи с задачей Нехари предъявили некоторое условие того, что оператор из $B\left(\mathbb{H}^{2}\right)$ является оператором Ганкеля. Однако удобного условия до сих пор не существовало. Дадим такое условие в следующем виде.

Пусть $U$ - тёплицев оператор $T_{R_{\delta}}$. Тогда $U$ - изометрия из $B\left(\mathbb{H}^{2}\right)$ и справедлива следующая теорема.

Tеорема 3 (ср. [2, Theorem 3.5]). Пусть $H \in B\left(\mathbb{H}^{2}\right)$. Тогда следующие условия эквивалентны:

(i) $H$ является оператором Ганкеля, т.е. существует такой элемент $A$ из $\mathfrak{L}$, что $H=H_{A}$;

(ii) $U^{*} H=H U$ u WHP принадлежит коммутанту алгебры $R(M)$;

(iii) $H$ имеет матричное представление вида $\left(\ell_{x_{-i-j-1}} u^{-i-j-1}\right)_{i, j=0}^{\infty}$, где $x_{k} \in$ $M$ для всех $k \leqslant-1$.

При любом из этих условий

$$
\|H\|=\inf \left\{\|A\| \mid A \in \mathfrak{L} u H_{A}=H\right\} .
$$

Доказательство. Эквивалентность (i) $\Leftrightarrow$ (iii) вытекает из [2, Theorem 3.5].

(i) $\Rightarrow$ (ii). Предположим, что существует такой оператор $A$ из $\mathfrak{L}$, что $H=H_{A}$. Поскольку $W H P=(I-P) A P$, ясно, что $W H P \in R(M)^{\prime}$. Так как $R_{\delta} \mathbb{H}^{2} \subseteq \mathbb{H}^{2}$, имеем

$$
\begin{aligned}
U^{*} H & =U^{*} H_{A}=P R_{\delta}^{*} P W(I-P) A P=P R_{\delta}^{*}(P W(I-P)) A P \\
& =P(P W(I-P)) R_{\delta}^{*} A P=P W(I-P) A R_{\delta} P \\
& =P W(I-P) A P R_{\delta} P=H_{A} U=H U .
\end{aligned}
$$

(ii) $\Rightarrow$ (iii). Если выполнено (ii), то, согласно предложению 2, WHP имеет матричное представление $\left(\ell_{x_{i j}} u^{i-j}\right)_{i, j=-\infty}^{\infty}, x_{i j} \in M$, где $x_{i j}=0$ при $(i, j) \notin$ $\mathbb{Z}_{-} \times \mathbb{Z}_{+}$. Таким образом, $W H P$ имеет вид $\left(\ell_{x_{-i-1} j} u^{-i-j-1}\right)_{i, j=-\infty}^{\infty}$, где $x_{-i-1 j}=$ 0 при $(m, n) \notin \mathbb{Z}_{+}^{2}$. Поскольку $U^{*} H=H U$, то для любых $i, j \geqslant 0$ имеем $\ell_{x_{-i-1}{ }_{j}} u^{-i-j-1}=\ell_{x_{-i-2} j+1} u^{-i-j-1}$ и потому $x_{-i-1 j}=x_{-i-2 j+1}$. Отсюда следует, что $H$ имеет требуемое матричное представление. Теорема доказана.

По аналогии с теоремой 3 можно получить следующий результат.

Теорема 4. Пусть $H \in B\left(\mathbb{H}^{2}\right)$. Тогда следующие условия эквивалентны:

(i) существует такой элемент $A$ из $\mathfrak{L}$, что $H=\widetilde{H}_{W A W}$;

(ii) $U^{*} H=H U$ и $H P W$ принадлежит коммутанту алгебры $R(M)$.

При этих условиях

$$
\|H\|=\inf \left\{\|A\| \mid A \in \mathfrak{L} u \widetilde{H}_{W A W}=H\right\} .
$$

Простыми вычислениями можно доказать следующие леммы.

Лемма 5. Для любого $А$ из $\mathfrak{L}$

(i) $H_{A}=0$ тогда и только тогда, когда $A$ лежит в $\mathfrak{L}_{+}$; 
(ii) $\widetilde{H}_{W A W}=0$ тогда и только тогда, когда $A^{*}$ лежит в $\mathfrak{L}_{+}$.

Лемма 6. Если $A$ и $B$ принадлежат $\mathfrak{L}$, mo $\left(H_{A}\right)^{*} H_{B}=T_{A^{*} B}-T_{A^{*}} T_{B}$.

Лемма 7. Оператор $W A^{*} W \quad \mathbb{H}^{2}$-инвариантен для любого $A$ из $\mathfrak{L}_{+}$.

Для любого $A$ из $\mathfrak{L}$ положим $\widetilde{T}_{W A W}=P W A W P$. Справедлива следующая лемма.

Лемма 8. Для любых $A \in \mathfrak{L} u B \in \mathfrak{L}_{+}$

(i) $H_{A} T_{B}=H_{A B}$;

(ii) $\widetilde{H}_{W A W} \widetilde{T}_{W B^{*} W}=\widetilde{H}_{W A B^{*} W}$.

Теперь мы можем доказать основной результат.

ДОКАЗАТЕЛЬСТВО ТЕОРЕМЫ 1. Поскольку $V$ унитарен, по лемме 6 получаем

$$
\left(H_{V^{*}}\right)^{*} H_{V^{*}}=T_{V V^{*}}-T_{V} T_{V^{*}}=I-T_{V} T_{V^{*}} .
$$

Поэтому $\left(H_{V^{*}}\right)^{*} \mathbb{H}^{2}=\mathscr{K}$. Так как $\left(H_{V^{*}}\right)^{*}=\widetilde{H}_{W V W}$, то $U^{*}\left(H_{V^{*}}\right)^{*}=\left(H_{V^{*}}\right)^{*} U$ и $\left(H_{V^{*}}\right)^{*} P W$ принадлежит коммутанту алгебры $R(M)$ по теореме 4 . При этом $U=P_{\mathscr{K}} R_{\delta} P_{\mathscr{K}}$ на $\mathscr{K}$. Таким образом,

$$
U^{*}\left(A^{*}\left(H_{V^{*}}\right)^{*}\right)=A^{*} U^{*}\left(H_{V^{*}}\right)^{*}=\left(A^{*}\left(H_{V^{*}}\right)^{*}\right) U .
$$

Для любого $x$ из $M$ имеем

$$
R_{x}\left(A^{*}\left(H_{V^{*}}\right)^{*} P W\right)=A^{*} R_{x}\left(H_{V^{*}}\right)^{*} P W=\left(A^{*}\left(H_{V^{*}}\right)^{*} P W\right) R_{x} .
$$

Итак, $A^{*}\left(H_{V^{*}}\right)^{*} P W \in R(M)^{\prime}$ и, следовательно, $A^{*}\left(H_{V^{*}}\right)^{*}$ удовлетворяет условию (ii) теоремы 4. Поэтому можно выбрать такое $D \in \mathfrak{L}$, что $A^{*}\left(H_{V^{*}}\right)^{*}=$ $\widetilde{H}_{W D W}$ и $\left\|\widetilde{H}_{W D W}\right\|=\|D\|$. Положим $C=V D^{*}$; тогда $C$ лежит в $\mathfrak{L}$. По лемме 8 имеем

$$
\begin{aligned}
H_{C} & =H_{V D^{*}}=\left(\widetilde{H}_{W D V^{*} W}\right)^{*}=\left(\widetilde{H}_{W D W} \widetilde{T}_{W V^{*} W}\right)^{*}=\left(A^{*}\left(H_{V^{*}}\right)^{*} \widetilde{T}_{W V^{*} W}\right)^{*} \\
& =\left(\widetilde{T}_{W V^{*} W}\right)^{*} H_{V^{*}} A=\left(\widetilde{T}_{W V^{*} W}\right)^{*}\left(\widetilde{H}_{W V W}\right)^{*} A=\left(\widetilde{H}_{W V W} \widetilde{T}_{W V^{*} W}\right)^{*} A \\
& =\left(\widetilde{H}_{W V V^{*} W}\right)^{*} A=\left(H_{I}\right)^{*} A=0,
\end{aligned}
$$

поскольку $I \in \mathfrak{L}_{+}$. Поэтому $C \in \mathfrak{L}_{+}$в силу леммы 5 , и мы замечаем, что $D^{*}=V^{*} C$. Тогда

$$
A^{*}\left(H_{V^{*}}\right)^{*}=\widetilde{H}_{W D W}=\left(H_{D^{*}}\right)^{*}=\left(H_{V^{*} C}\right)^{*}=\left(H_{V^{*}} T_{C}\right)^{*}=\left(T_{C}\right)^{*}\left(H_{V^{*}}\right)^{*}
$$

по лемме 8. Итак, $A^{*}=\left(T_{C}\right)^{*}$ на $\mathscr{K}$. Поэтому

$$
A=P_{\mathscr{K}} T_{C} P_{\mathscr{K}}=P_{\mathscr{K}} C P_{\mathscr{K}}=S_{C} \text {. }
$$

Отсюда следует, что коммутант алгебры $P_{\mathscr{K}} \mathfrak{R}_{+} P_{\mathscr{K}}$ содержится в $P_{\mathscr{K}} \mathfrak{L}_{+} P_{\mathscr{K}}$. Обратное включение тривиально, так что $\left(P_{\mathscr{K}} \mathfrak{R}_{+} P_{\mathscr{K}}\right)^{\prime}=P_{\mathscr{K}} \mathfrak{L}_{+} P_{\mathscr{K}}$.

По формуле (1) имеем

$$
\|A\|=\left\|P_{\mathscr{K}} C P_{\mathscr{K}}\right\| \leqslant\|C\| .
$$

С другой стороны,

$$
\|C\|=\|D\|=\left\|\widetilde{H}_{W D W}\right\|=\left\|A^{*}\left(H_{B^{*}}\right)^{*}\right\| \leqslant\|A\| .
$$

Поэтому $\|A\|=\|C\|$. Теорема доказана.

Благодарность. Автор чрезвычайно признателен рецензенту за множество полезных замечаний и предложений. 


\section{ЛиТЕРАТУРА}

1. Haagerup U. Math. Scand., 37, 271-283 (1975). 2. Imina Y., Saito K.-S. Canad. Math. Bull., 37, 75-81 (1994). 3. McAsey M., Muhly P. S., Saito K.-S. Trans. Amer. Math. Soc., 248, 381-409 (1979). 4. Ohwada T. The adjoint operators of Hankel operators associated with analytic crossed products. Preprint. 5. Saito K.-S. Math. Proc. Camb. Phil. Soc., 108, 539-549 (1990). 6. Saito K.-S. Math. Proc. Camb. Phil. Soc., 117, 1120 (1995). 7. Sarason D. Trans. Amer. Math. Soc., 127, 179-203 (1967). 8. Yoshino T. Yokohama Math. J., 44, 21-24 (1997).

Department of General Science Tsuruoka National College of Technology

Tsuruoka, Japan

e-mail: ohwada@tsuruoka-nct.ac.jp
Поступило в редакцию 11 марта 2004 г. 